\title{
Características de salud mental de los hombres que maltratan a su pareja
}

\author{
E Calvete
}

Universidad de Deusto. Departamento de Psicología

\begin{abstract}
RESUMEN
La prevalencia de trastornos psicopatológicos en los hombres que maltratan a sus parejas íntimas es un tema aún no resuelto. En este artículo se revisan los estudios realizados sobre las características de salud mental de los maltratadores. La mayoría de estos estudios se ha basado en muestras de maltratadores que recibían tratamiento o que estaban en prisión. Por lo general evalúan la presencia de trastornos psicopatológicos mediante autoinformes, empleándose en pocas ocasiones entrevistas diagnósticas. Los resultados de estas investigaciones muestran que los maltratadores tienden a obtener puntuaciones altas en algunos trastornos de personalidad, especialmente en los tipos narcisista, antisocial y "borderline”. También presentan a menudo trastornos depresivos y consumo de alcohol y drogas. Además, algunos estudios encuentran que los problemas neurológicos son relativamente frecuentes. Finalmente se discuten las limitaciones de las investigaciones realizadas y las implicaciones para el tratamiento de maltratadores.
\end{abstract}

Palabras clave: Maltrato Conyugal, Psicopatología, Trastornos de Personalidad, Depresión, Alcoholismo, Trastornos Relacionados con Sustancias.

\section{MENTAL HEALTH CHARACTERISTICS OF MEN WHO ABUSE THEIR INTIMATE PARTNER}

\begin{abstract}
The prevalence of psychopathological disorders amongst men who abuse their intimate partners has yet to be established. This article reviews studies carried out to ascertain the mental health characteristics of male domestic abusers. Most of these studies are based on samples of abusers under treatment or in prison. They generally assess the presence of psychopathological disorders through self-reports and diagnostic interviews are infrequently used. The results of this research show that domestic abusers tend to obtain high points for some types of personality disorders, especially narcissistic, antisocial and borderline disorders. They also present symptoms of depressive disorders and consumption of drugs and alcohol. Some studies also show that neurological problems are relatively frequent. Finally I discuss the limitations of current research and the implications for treatment of domestic abusers
\end{abstract}

Key Words: Spouse Abuse, Psychopathology, Personality Disorders, Depression, Alcoholism, Substance Related Disorders.

Texto recibido: mayo 2008

Texto aceptado: junio 2008

La violencia contra las mujeres es uno de los problemas más graves de nuestra sociedad. Adopta múltiples modalidades incluyendo el maltrato psicológico, acoso, abuso sexual y maltrato físico, llegando en su extremo más cruel al homicidio'. Sólo en 2007, en España 72 mujeres fueron asesinadas por sus parejas. Los datos nos indican además que el problema pre- senta una tendencia creciente. Por ejemplo, la comparación de dos estudios realizados en 1999 y 2006 ha mostrado que las tasas de prevalencia en población general han aumentado del 8,05 al 17,9\% para agresión física y del 11,48 al 30,1\% para abuso sexual2-3.

El maltrato tiene graves consecuencias para la salud física y mental de las víctimas, llevando al desarro- 
llo de numerosos problemas tales como el trastorno por estrés postraumático, depresión, ansiedad, y consumo de alcohol y drogas. Además, a menudo se produce un solapamiento entre la violencia contra la mujer y la violencia contra los hijos, estimándose que en torno al 50\% de los casos éstos son también víctimas directas. En el resto de los casos son testigos de la violencia, aspecto que puede dañar seriamente su desarrollo afectivo ${ }^{4}$.

Lo anterior ha motivado que el estudio de la violencia contra las mujeres haya experimentado un aumento extraordinario en las dos últimas décadas. La mayoría de las investigaciones se han centrado en el impacto de la violencia sobre las víctimas, con relativamente muchos menos estudios centrados en el maltratador. Sin embargo, desde una perspectiva preventiva resulta fundamental la identificación de los factores que contribuyen a que un hombre actúe de forma violenta contra su pareja. Esto ha llevado a plantear la hipótesis de que los maltratadores podrían presentar determinados rasgos psicopatológicos que les pondrían en riesgo de ejercer la violencia contra sus parejas. En este artículo se revisan los principales estudios realizados sobre las características de salud mental de los maltratadores.

\section{TIPOS DE MALTRATADORES}

La presencia de problemas de salud mental ha sido precisamente uno de los criterios que se han empleado en la mayoría de las tipologías de maltratadores. Probablemente la tipología más citada es la que desarrollaron Holzworth-Munroe y Stuart ${ }^{5}$, en la que basándose en tres dimensiones (severidad de la violencia, generalidad, y presencia de psicopatología o trastornos de personalidad) propusieron la existencia de tres tipos de maltratadores:

El primer tipo se caracteriza por ejercer la violencia solamente en la familia e incluye maltratadores que se muestran como menos desviados en algunos indicadores tales como impulsividad, consumo de alcohol y drogas y conducta delincuente. Suelen tener dificultades en cuanto a habilidades sociales y comunicación, un historial de exposición a la agresión en la familia de origen y altos niveles de dependencia de su pareja. Sus relaciones de pareja suelen ser relativamente estables y en comparación con los demás tipos muestran mayores niveles de remordimiento tras el acto violento. Constituyen aproximadamente el $50 \%$ de los maltratadores.

El segundo tipo fue denominado como borderline/disfórico. Tienden a involucrarse en violencia de moderada a severa. Presentan problemas emocionales, especialmente de control de la ira y de celos. Suelen caracterizarse por una historia de rechazo por parte de los padres, abuso infantil, alta dependencia de su pareja, habilidades sociales y de comunicación pobres, hostilidad hacia las mujeres y bajo nivel de arrepentimiento por la violencia ejercida.

El tercer tipo es el de los maltratadores generalmente violentos/antisociales. Éstos suelen ejercer violencia de moderada a severa, física, psicológica y sexual. A menudo han sufrido violencia en su familia de origen y suelen presentar un historial de delincuencia. También son característicos los déficits en habilidades sociales y las creencias justificadoras de la violencia, como una respuesta apropiada a la provocación. Según Holzworth-Munroe y Stuart ${ }^{5}$, en este grupo son habituales los trastornos de personalidad antisocial y narcisista.

Estudios posteriores han mostrado que esta tipología no explica todos los casos. Por ejemplo, el mismo equipo de investigadores identificó un cuarto grupo de maltratadores que denominó antisocial de bajo nivel, constituyendo el $33 \%$ de la muestra de dicho estudio $^{6}$.

La tipología de Holzworth-Munroe y Stuart fue fundamentalmente teórica, pero posteriormente se han desarrollado otras muy similares mediante el empleo de métodos empíricos. De esta manera, por ejemplo, se ha diferenciado entre maltratadores sin rasgos patológicos, maltratadores dependientes pasivo-agresivos y maltratadores antisociales ${ }^{7}$, y entre maltratadores no patológicos (coincidente con el violento sólo en la familia), tipo patológico (coincidente con el disfórico/borderline) y tipo psicopático o antisocial ${ }^{8}$.

Por tanto, tipologías como las mencionadas destacan rasgos psicopatológicos como elementos diferenciadores de algunos tipos de maltratadores. En particular la mayoría hace referencia a trastornos de la personalidad (TP) y a otras dificultades psicológicas como la depresión, ansiedad y consumo abusivo de sustancias. La Tabla I presenta una síntesis de numerosos estudios sobre la presencia de estos problemas en los maltratadores ${ }^{9-22}$, y en los siguientes apartados se describen los hallazgos más relevantes sobre los mismos.

\section{TRASTORNOS DE PERSONALIDAD}

La mayoría de los estudios que han evaluado la psicopatología de los maltratadores se han centrado en los trastornos de personalidad (TP), los cuales es- 


\begin{tabular}{|c|c|c|c|}
\hline & Muestras & Medidas & Resultados para los maltratadores \\
\hline Beasley y Stoltenberg $(1992)^{9}$ & $\begin{array}{l}49 \text { hombres abusadores y } 35 \\
\text { hombres no abusadores pero } \\
\text { implicados en relaciones de } \\
\text { pareja conflictivas }\end{array}$ & $\begin{array}{l}\text { MCMI-II } \\
\text { Inventario de } \\
\text { Personalidad } \\
\text { Narcisista } \\
\text { STAI }\end{array}$ & $\begin{array}{l}\text { Puntuaciones mayores en narcisismo, perso- } \\
\text { nalidad antisocial, esquizotípica, borderline y } \\
\text { agresiva/sádica, y en ansiedad estado y rasgo. }\end{array}$ \\
\hline Belfrage y Rying $(2004)^{10}$ & $\begin{array}{l}164 \text { maltratadores que co- } \\
\text { metieron homicidio }\end{array}$ & $\begin{array}{l}\text { Entrevista para } \\
\text { evaluar } \\
\text { criterios } \\
\text { DSM-IV } \\
\text { Escala de } \\
\text { psicopatía } \\
\text { PCL-SV }\end{array}$ & $\begin{array}{l}\text { Todos los casos menos } 5 \text { fueron diagnostica- } \\
\text { dos con al menos un trastorno: } 36 \% \text { psicosis, } \\
3 \% \text { trastorno distímico, } 11 \% \text { depresión ma- } \\
\text { yor, } 2 \% \text { abuso de sustancias } \\
\text { TP: } 1 \% \text { paranoide, } 5 \% \text { antisocial, } 4 \% \text { border- } \\
\text { line, } 1 \% \text { histriónica, } 6 \% \text { narcisista, } 15 \% \text { no } \\
\text { especificada }\end{array}$ \\
\hline Boyle y Vivian $(1996)^{11}$ & $\begin{array}{l}263 \text { hombres en terapia de } \\
\text { pareja ( } 94 \text { no violentos, } 69 \\
\text { moderadamente violentos, y } \\
100 \text { severamente violentos). } \\
\text { Un grupo de comparación de } \\
\text { la comunidad compuesto por } \\
\text { hombres satisfechos en sus } \\
\text { relaciones de pareja }\end{array}$ & & $\begin{array}{l}\text { Los severamente violentos mostraron mayo- } \\
\text { res niveles de ira, síntomas depresivos }\end{array}$ \\
\hline $\begin{array}{l}\text { Else, Wonderlich, Beatty y } \\
\text { Christie }(1993)^{12}\end{array}$ & $\begin{array}{l}21 \text { maltratadores y } 21 \text { no } \\
\text { maltratadores }\end{array}$ & MMPI y PDS & $\begin{array}{l}\text { Los maltratadores puntaron más alto en TP } \\
\text { borderline y antisocial } \\
\text { No hubo diferencias en síntomas de depresión } \\
\text { ni en consumo de alcohol }\end{array}$ \\
\hline Flournoy y Wilson $(1991)^{13}$ & $\begin{array}{l}56 \text { maltratadores en trata- } \\
\text { miento }\end{array}$ & MMPI & $\begin{array}{l}\text { Se caracterizaron por tendencia a adicciones y } \\
\text { a depresión }\end{array}$ \\
\hline $\begin{array}{l}\text { Gavazzi, Julian, y McKenry } \\
(1996)^{14}\end{array}$ & $\begin{array}{l}152 \text { a los que se evaluó el uso } \\
\text { de la violencia contra la pa- } \\
\text { reja }\end{array}$ & $\begin{array}{l}\text { BSI (Brief } \\
\text { Symptom } \\
\text { Inventory) }\end{array}$ & $\begin{array}{l}\text { Los violentos mostraron mayores puntuacio- } \\
\text { nes en las subescalas de depresión, ansiedad } \\
\text { general y fóbica, hostilidad, ideación paranoi- } \\
\text { de, sensibilidad interpersonal y psicoticismo }\end{array}$ \\
\hline Gondolf $(1999)^{15}$ & $\begin{array}{l}840 \text { maltratadores remitidos } \\
\text { judicialmente a tratamiento }\end{array}$ & MCMI-III & $\begin{array}{l}11 \% \text { depresión mayor } \\
40 \% \text { trastornos de ansiedad } \\
90 \% \text { puntuó positivo para al menos un TP } \\
(25 \% \text { narcisista, } 24 \% \text { pasivo-negativo, } 19 \% \\
\text { antisocial, y } 19 \% \text { depresiva) } \\
26 \% \text { había recibido tratamiento en el pasado } \\
\text { para alcoholismo o consumo de drogas }\end{array}$ \\
\hline Grann y Wedin $(2002)^{16}$ & 88 maltratadores en prisión & $\begin{array}{l}\text { PCL-R y otras } \\
\text { medidas }\end{array}$ & $\begin{array}{l}51 \% \text { abuso o dependencia de alcohol o dro- } \\
\text { gas } \\
27 \% \text { psicopatía }\end{array}$ \\
\hline $\begin{array}{l}\text { Hamberger y Hastings } \\
(1991)^{17}\end{array}$ & $\begin{array}{l}38 \text { maltratadores alcohólicos } \\
61 \text { maltratadores no alcohó- } \\
\text { licos } \\
92 \text { hombres de la comunidad, } \\
\text { de los cuales } 28 \text { fueron iden- } \\
\text { tificados como violentos }\end{array}$ & MCMI & $\begin{array}{l}\text { Los maltratadores puntuaron más alto en tras- } \\
\text { torno de personalidad límite, especialmente } \\
\text { los que además eran alcohólicos }\end{array}$ \\
\hline $\begin{array}{l}\text { Hanson, Cadsky, Harris, } \\
\text { Lalonde }(1997)^{18}\end{array}$ & $\begin{array}{l}997 \text { divididos según una } \\
\text { prueba de autoinforme en no } \\
\text { maltratadores (184), maltra- } \\
\text { tadores moderados (517) y } \\
\text { maltratadores severos (296) }\end{array}$ & $\begin{array}{l}\text { Cuestionario } \\
\text { con múltiples } \\
\text { medidas. }\end{array}$ & $\begin{array}{l}\text { Los hombres que puntuaron alto en conduc- } \\
\text { ta abusiva se caracterizaron en mayor medida } \\
\text { por TP antisocial, síntomas de estrés, y con- } \\
\text { sumo de sustancias }\end{array}$ \\
\hline
\end{tabular}




\begin{tabular}{|c|c|c|c|}
\hline & Muestras & Medidas & Resultados para los maltratadores \\
\hline $\begin{array}{l}\text { Hart, Dutton, y Newlove } \\
(1993)^{19}\end{array}$ & $\begin{array}{l}85 \text { maltratadores voluntarios } \\
\text { y remitidos judicialmente }\end{array}$ & $\begin{array}{l}\text { MCMI-II, } \\
\text { entrevista }\end{array}$ & $\begin{array}{l}\text { La entrevista mostró una prevalencia del 50\% } \\
\text { para TPs. El MCMI-II indicó que el } 80-90 \% \\
\text { presentaban algún TP. Destacaron los TP nar- } \\
\text { cisista }(58 \%) \text { y antisocial }(60 \%)\end{array}$ \\
\hline Sugihara y Warner $(1999)^{20}$ & $\begin{array}{l}60 \text { maltratadores y } 45 \text { no mal- } \\
\text { tratadores de la comunidad }\end{array}$ & MCMI-III & $\begin{array}{l}\text { Los maltratadores puntuaron más alto en nu- } \\
\text { merosos TP, siendo las diferencias mayores en } \\
\text { personalidad paranoide, esquizoide, esquizo- } \\
\text { típico }\end{array}$ \\
\hline
\end{tabular}

\section{Estudios en España}

\begin{tabular}{|c|c|c|c|}
\hline $\begin{array}{l}\text { Echeburua, Fernández- } \\
\text { Montalvo y Amor }(2003)^{21}\end{array}$ & 54 maltratadores en prisión & SCL-90-R & $\begin{array}{l}\text { Niveles de síntomas psicopatológicos simila- } \\
\text { res a los de la población general } \\
\text { Más frecuentes los antecedentes psiquiátricos } \\
\text { entre los que no habían intentado homicidio }\end{array}$ \\
\hline $\begin{array}{l}\text { Echeburua y Fernández- } \\
\text { Montalvo }(2007)^{22}\end{array}$ & 162 maltratadores en prisión & SCL-90-R & $\begin{array}{l}12 \% \text { mostraron probable psicopatía } \\
71 \% \text { tenían historia psiquiátrica previa }\end{array}$ \\
\hline
\end{tabular}

Nota. MCMI $=$ The Millon Clinical Multiaxial Inventory, MMPI $=$ The Minnesota Multiphasic Personality Inventory, BSI $=$ the Brief Symptom Inventory, SCL-90-R = The Symptoms Check List-90-Revised, PCL-R = Hare's Psychopathy Checklist-Revised.

Tabla I. Descripción de algunos estudios que han evaluado las características de salud mental de los maltratadores.

tán encuadrados en el Eje II del DSM-IV-R ${ }^{23}$. Estos trastornos habitualmente tienen su origen en la infancia y se describen como patrones de percepción y relación con el mundo relativamente crónicos, generalizados y rígidos. Como resultado, las personas con estos trastornos a menudo se relacionan con los demás de forma disfuncional. Estos individuos no suelen buscar ayuda de forma espontánea y cuando lo hacen generalmente es debido a que se les impone.

Tal y como puede observarse en la Tabla I, los TP más identificados en maltratadores son el tipo borderline, consistente en inestabilidad general en las relaciones interpersonales, autoimagen y afectividad, así como impulsividad, el tipo narcisista, caracterizado por grandiosidad, necesidad de admiración y falta de empatía, el tipo antisocial, caracterizado por desprecio y violación de los derechos de los demás, y el tipo paranoide, que implica un carácter rencoroso, desconfiado y la tendencia a reaccionar con ira y agresividad.

Ferrer y colaboradores en un estudio meta-analítico revisaron las investigaciones realizadas entre 1988 y 1998 concluyendo que si bien los maltratadores puntúan más alto en una gran variedad de TP las diferencias respecto a otros colectivos son pequeñas ${ }^{24}$. Con posterioridad al periodo evaluado por Ferrer y colaboradores se han publicado nuevos trabajos. Uno de los más importantes es el de Gondolf, quien eva- luó a 840 maltratadores remitidos judicialmente a tratamiento y los comparó con otras dos muestras $(600$ pacientes psiquiátricos y 100 personas en tratamiento por consumo de drogas) ${ }^{15}$. Según sus resultados, el 90\% de los maltratadores presentaría características de personalidad clínica, destacando el tipo narcisista $(25 \%)$, negativista $(24 \%)$, antisocial $(19 \%$,) y depresivo $(19 \%)$. Sin embargo, sólo el $49 \%$ mostró puntuaciones suficientemente altas para indicar trastorno de personalidad. Además, en contraste con otros estudios, Gondolf encontró que relativamente pocos maltratadores puntuaban alto en TP borderline (6\%).

El estudio de Gondolf, al igual que la mayoría de los presentados en la Tabla I, han sido criticados por basarse en autoinformes ${ }^{25}$. Empleando una metodología diferente para la evaluación de los trastornos de personalidad, Belfrage y Rying estudiaron los $162 \mathrm{ca}-$ sos de homicidio contra la pareja que habían tenido lugar en Suecia en el periodo 1990-199910. Revisaron los informes forenses existentes, aplicaron nuevas medidas y realizaron entrevistas cuando fue posible (en su estudio el $24 \%$ de los casos se habían suicidado tras el homicidio). Las tasas de TP que encontraron fueron mucho más modestas (véase Tabla I), siendo los más característicos el tipo narcisista y antisocial.

Por último, para cerrar este apartado, hay estudios que se han centrado exclusivamente en los rasgos de personalidad psicopática, asociados fundamen- 
talmente al tipo antisocial de maltratador ${ }^{26-27}$. Estos rasgos incluyen características conductuales y de estilo de vida, tales como impulsividad y conductas antisociales, junto con características interpersonales y afectivas, tales como la falta de remordimiento y empatía, el egocentrismo y la manipulación ${ }^{10}$. Hay varios estudios realizados en prisiones sobre psicopatía en maltratadores, pero resultan poco consistentes en cuanto a las tasas obtenidas. Por ejemplo, Echeburua y Fernández-Montalvo ${ }^{22}$ encontraron una tasa del $12 \%$, muy por debajo de la obtenida en otros estu$\operatorname{dios}^{16}$. Además, se ha encontrado que los maltratadores antisociales en prisión difieren de otros delincuentes antisociales en prisión en algunos de los rasgos de psicopatía. En concreto parecen caracterizarse por una experiencia afectiva deficiente y por menos impulsividad e irresponsabilidad ${ }^{27}$.

\section{DEPRESIÓN Y ANSIEDAD}

La literatura también muestra que los maltratadores pueden presentar en mayor medida que la población general trastornos psicopatológicos del Eje I del DSM-IV. Entre éstos, destacarían los trastornos depresivos, los cuales serían coherentes sobre todo con el tipo de maltratador borderline/disfórico ${ }^{26}$.

En el mencionado estudio de Gondolf, el 20\% de los maltratadores presentaron trastornos mentales severos pertenecientes al Eje I, y esto sin tener en cuenta los trastornos de ansiedad y dependencia de alcohol y drogas ${ }^{15}$. El trastorno más característico fue la depresión mayor (11\% de la muestra total). En opinión de Gondolf la depresión no parecía ser una consecuencia de los últimos incidentes (asalto, detención) ya que se encontró que el $31 \%$ de los que estaban deprimidos habían intentado suicidarse o habían amenazado con hacerlo en el pasado. De hecho, se observó que el $22 \%$ de la muestra había recibido en el pasado algún tipo de tratamiento para sus problemas de salud mental ( $8 \%$ medicación, $16 \%$ terapia psicológica, $6 \%$ hospitalización psiquiátrica) ${ }^{28}$. La tasa de prevalencia de depresión mayor que encontró Gondolf es similar a la encontrada en otro estudio basado en hombres acusados de homicidio contra su pareja ${ }^{10}$.

Gondolf no contabilizó los casos de ansiedad en la estadística mencionada sobre trastornos del Eje I porque pensó que ésta, presente en el $40 \%$ de los casos, podía ser reactiva ante el hecho de haber sido arrestado y remitido a un programa para maltratadores. Otras investigaciones también han observado mayores puntuaciones en ansiedad entre los maltratadores $^{9,24}$.

\section{CONSUMO DE ALCOHOL Y DROGAS}

Numerosos estudios coinciden en el hallazgo de tasas altas de consumo de alcohol y drogas en los maltratadores ${ }^{29}$. Por ejemplo, Gondolf encontró en su muestra de maltratadores que el $26 \%$ había recibido tratamiento en el pasado para alcoholismo o consumo de drogas ${ }^{15}$. Grann y Wedin evaluaron a 88 reclusos cumpliendo condena por homicidio o agresión a su pareja y encontraron que el $51 \%$ presentaba un problema de abuso o dependencia de alcohol o drogas $(31 \%$ solo alcohol, $5 \%$ alcohol y otra droga, y $16 \%$ múltiples sustancias) ${ }^{16}$.

Además, el consumo de alcohol y drogas parece vincularse directamente con los acontecimientos violentos $^{30,31}$. Por ejemplo, Sharps y colaboradores encontraron que dos tercios de los maltratadores acusados de homicidio o de intento de homicidio habían abusado del alcohol, drogas o ambos antes del incidente $^{32}$. En otro estudio, Fals-Stewart examinó registros diarios de parejas con historial de violencia durante 15 meses y encontró que la probabilidad de un episodio severo de violencia era más de 11 veces mayor en los días en los que el hombre había consumido alcohol. Además el $60 \%$ de los episodios tuvieron lugar dentro de las dos horas de bebida ${ }^{31}$.

El consumo de sustancias tales como barbitúricos, anfetaminas, opiáceos, cocaína y combinaciones alcohol/coca también se ha asociado con la violencia. En particular, el consumo de cocaína está recibiendo una gran atención. Logan, Walker, Staton y Lenkefeld $^{33}$ dividieron a 500 reclusos según el grado de violencia ejercida contra sus parejas (leve, moderada, extrema) y mediante entrevistas evaluaron el consumo de sustancias. Encontraron que aquellos en el grupo de violencia extrema informaban de más años de consumo regular de cocaína (4,5 años) que los del grupo de violencia moderada (2,9 años) o de baja violencia (2,6 años). Logan y colaboradores sugirieron varias explicaciones alternativas para la asociación entre cocaína y violencia. Por ejemplo, la cocaína puede causar violencia a través de sus efectos farmacológicos, o puede agravar y potenciar un temperamento agresivo y hostil, contribuyendo a la expresión de la violencia, o, por último, puede que el consumo de cocaína y la violencia sean simplemente dos manifestaciones de la conducta antisocial.

\section{PROBLEMAS NEUROLÓGICOS}

Un tema relativamente poco explorado es el referente a la presencia de déficits neurológicos en los 
maltratadores ${ }^{34}$. Por ejemplo, Rosenbaum y colaboradores compararon una muestra de 53 maltratadores con otra de 45 hombres con relaciones de pareja satisfactorias y 32 con relaciones conflictivas ${ }^{35}$. Encontraron que el daño cerebral era un rasgo asociado significativamente a los maltratadores, ya que la tasa de prevalencia de daño cerebral fue considerablemente mayor en el grupo de maltratadores que en los otros dos grupos. En concreto el 83,79 \% del grupo de maltratadores presentó daño cerebral y éste fue clínico en el 52,83\% de los maltratadores. Pudieron mostrar además que en el 93,1\% de los casos de maltratador con daño cerebral, el daño precedía al abuso. En otro estudio posterior se encontró que el $48 \%$ de una muestra de maltratadores presentaba trastornos neuropsicológicos, porcentaje muy superior al que presentó el grupo control $(4,3 \%)^{36}$.

Ciertos déficits neurológicos y neuropsicológicos (especialmente los de los lóbulos frontales y/o temporales) pueden aumentar la probabilidad de expresión de actos violentos de naturaleza impulsiva. Una interpretación de este tipo de hallazgos es que el déficit neurológico podría reducir la capacidad de controlar los impulsos, que en caso de ser de naturaleza violenta podrían desencadenar el acto agresivo. De hecho, un estilo impulsivo de conducta combinado con determinados rasgos cognitivos es uno de los factores de riesgo más consistentes de conducta violen$\mathrm{ta}^{37}$. Sin embargo, son también posibles otras interpretaciones. Un estilo impulsivo previo podría aumentar tanto el riesgo de un daño cerebral, por ejemplo a través de una mayor propensión a los accidentes, como el riesgo de conducta violenta. También el consumo de alcohol podría ser el factor que lleva tanto a riesgo de maltrato como de lesión cerebral, por ejemplo, a través de conducción temeraria.

\section{CONCLUSIONES E IMPLICACIONES PARA EL TRATAMIENTO}

La revisión anterior nos muestra que el colectivo de maltratadores tiende a mostrar numerosos trastornos psicopatológicos, sin que haya una consistencia entre estudios en cuanto a la naturaleza y prevalencia de los mismos.

En general hay un cierto consenso en que los TP son relativamente frecuentes entre los maltratadores, destacando los TP narcisista y antisocial, que serían característicos del tipo de maltratador denominado antisocial. Sin embargo, las tasas de prevalencia varían considerablemente de un estudio a otro, probablemente debido a las diferentes estrategias de evalua- ción empleadas. A este respecto es importante clarificar que la mayoría de los estudios se basan en autoinformes (por ejemplo, el MCMI) y no llegan a utilizar una entrevista diagnóstica, por lo que los resultados pueden estar sesgados por factores diversos tales como la deseabilidad social, dificultades en la comprensión de los items, etc. ${ }^{25}$.

Por otro lado, conviene puntualizar que las prevalencias de TP obtenidas en muestras de maltratadores están dentro del rango de las obtenidas en población penitenciaria en general, ya que este tipo de trastornos se presenta entre los más frecuentes en este colectivo $^{38}$. Por ejemplo, en un reciente estudio López-Barrachina, Lafuente y García-Latas encontraron tasas de prevalencia para los TP narcisista y antisocial de 59,7 y $47,5 \%$, respectivamente ${ }^{39}$.

La revisión realizada también nos muestra que los trastornos depresivos son frecuentes, especialmente en el subtipo de maltratador disfórico/borderline, y que dichos trastornos no suelen ser en general una consecuencia de los acontecimientos de maltrato y detención, sino que tienen su origen anteriormente.

Por último, los problemas con el alcohol y otras drogas son frecuentes entre los maltratadores, pareciendo ejercer además un papel clave en el desencadenamiento de los actos violentos.

Es importante reseñar que la mayoría de los estudios disponibles se han realizado en Estados Unidos, siendo muy pocos los realizados en España. Sin embargo, el perfil psicopatológico de los maltratadores podría diferir dependiendo de circunstancias culturales. Por ejemplo, las características de salud mental asociadas a maltratadores en Suecia son considerablemente diferentes a las encontradas en Estados Unidos $^{10}$. Esto alerta sobre la necesidad de realizar más investigación en nuestro contexto.

A pesar de las limitaciones, los resultados de las investigaciones disponibles tienen implicaciones clínicas. En concreto, el empleo de tipologías de maltratadores y la identificación de los rasgos psicopatológicos de éstos pueden ser de utilidad para determinar tanto la eficacia de tratamientos como el riesgo de futura reincidencia ${ }^{40}$.

Los maltratadores caracterizados por TP antisocial y rasgos psicopáticos presentan un mayor riesgo de reincidir ${ }^{16}$. Además responden peor a los tratamientos estándar para maltratadores ${ }^{8,26}$, probablemente debido a que su falta de arrepentimiento y de empatía con las víctimas implica muy poca motivación por un cambio de conducta. También el consumo de alcohol y drogas son factores asociados al abandono del programa de tratamiento ${ }^{8}$. En contraste, los maltratadores de tipo disfórico, que a menudo 
presentan sintomatología depresiva, tienden a estar mucho más motivados por recibir tratamiento y éste suele obtener un mejor resultado ${ }^{41}$.

El hecho de que la eficacia de los tratamientos dependa del tipo de maltratador ha llevado a desarrollar programas específicos. Por ejemplo, los maltratadores antisociales con rasgos psicopáticos parecen responder muy pobremente en contextos de terapia con grupos heterogéneos. El tratamiento de elección suele ser un grupo homogéneo con apoyo institucional o tratamiento individualizado de naturaleza cognitivo-conductual. En contraste, los abusadores cíclicos y emocionalmente inestables funcionan mejor en grupos heterogéneos y se benefician también de otros enfoques terapéuticos ${ }^{42}$.

Por último, esta revisión ha mostrado que los problemas neurológicos son relativamente frecuentes entre los maltratadores. Por ello, convendría evaluar su presencia en aquellos casos en los que se sospecha de algún tipo de daño, con el fin de incorporar en el tratamiento las estrategias de rehabilitación neuropsicológicas adecuadas ${ }^{36}$.

En síntesis, los maltratadores tienden a mostrar frecuentemente trastornos de personalidad y otros problemas psicopatológicos, sin que haya un consenso acerca de su prevalencia. El hecho de que la mayoría de los estudios haya tenido lugar en países anglosajones y que de hecho sean muy pocos los trabajos realizados en España, sugiere la conveniencia de nuevos estudios en nuestro entorno.

\section{REFERENCIAS BIBLIOGRÁFICAS}

1. Mahoney P, Williams LM, West C M. Violence against women by intimate relationship partners. En Renzetti CM, Edleson JL, Bergen RQ (Eds.). Sourcebook on violence against women; Thousand Oaks, CA: Sage; 2001. p. 143-178.

2. Medina-Ariza J, Barberet R. Intimate partner violence in Spain: Findings from a national survey. Violence Against Women 2003; 9: 302-22.

3. Calvete E, Corral S, Estévez A. Factor structure and validity of the Revised Conflict Tactics Scales for Spanish women. Violence Against Women 2007; 13: 1072-87.

4. Edleson JL. The overlap between child maltreatment and woman abuse. 1999 acceso 20 de diciembre de 2006]. Disponible en http: //www. vaw.umn.edu/documents/vawnet/overlap/overlap.html

5. Holtzworth-Munroe A, Stuart GL. Typologies of male batterers: Three subtypes and the differen- ces among them. Psychol Bull 1994 Nov; 116: 476-97

6. Holzworth-Munroe A, Meehan JC, Herron K, Rehman U, Stuart GL. Do subtypes of maritally violent men continue to differ over time? J Consult Clin Psychol 2003; 71: 728-40

7. Hamberger LK, Lohr JM, Borge D, Tolin DF. An empirical classification of motivations for domestic violence. Violence Against Women 1997; 3: 401-23.

8. Langhinrichsen-Rohling J, Huss MT, Ramsey S. The Clinical Utility of Batterer Typologies. J Fam Violence 2000; 15: 37-53.

9. Beasley R, Stoltenberg CD. Personality characteristics of male spouse abusers. Professional Psychology: Research \& Practice 1992; 23: 310-17.

10. Belfrage H, Rying M. Characteristics of spousal homicide perpetrators: a study of all cases of spousal homicide in Sweden 1990-1999.Crim Behav Ment Health 2004; 14: 121-33.

11. Boyle DJ, Vivian D. Generalized versus spousespecific anger/hostility and men's violence against intimates. Violence Vict 1996; 11: 293-317.

12. Else L, Wonderlich SA, Beatty WW, Christie DW, Staton RD. Personality characteristics of men who physically abuse women. Hosp Community Psychiatry 1993 Jan; 44: 54-8.

13. Flournoy PS, Wilson GL. Assessment of MMPI profiles of male batterers. Violence Vict 1991; 6: 309-20.

14. Gavazzi SM, Julian TW, McKenry PC. Utilization of the Brief Symptom Inventory to discriminate between violent and nonviolent male relationship partners. Psychological Reports 1996; 79: 1047-56.

15. Gondolf EW. MCMI-III results for batterer program participants in four cities: Less "pathological" than expected J. Fam. Violence 1999; 14: 1-17.

16. Grann M, Wedin I. Risk factors for recidivism among spousal assault and spousal homicide. Psychology, Crime and Law 2002; 8: 5-23.

17. Hamberger LK, Hastings JE. Personality correlates of men who batter and violent men: Some continuities and discontinuities. J Fam Violence 1991; 6: 131-47.

18. Hanson P K, Cadsky O, Harris A, Lalonde C. Correlates of battering among 997 men: Family history, adjustement, and attitudinal differences. Violence Vict 1997; 12: 191-208.

19. Hart S, Dutton D, Newlove T. The prevalence of personality disorders among wife assaulters. Personal. Dis 1993; 7: 329-41. 
20. Sugihara Y, Warner JA. Mexican-American male batterers on the MCMI-III. Psychol Rep. 1999; 85: 163-9.

21. Echeburúa E, Fernández-Montalvo J, Amor PJ. Psychopathological profile of men convicted of gender violence: A study in the prisons of Spain. J Interpers Violence 2003; 18: 798-812.

22. Echeburua E, Fernández-Montalvo J. Male batterers with and without psychopathy: an exploratory study in Spanish prisons. Int J Offender Ther Comp Criminol. 2007; 51: 254-63.

23. DSM-IV-R. Manual diagnóstico y estadístico de los trastornos mentales. Barcelona Masson, 2001.

24. Ferrer V, Bosch E, García E, Manassero MA, Gili M. Estudio Meta-Analítico de Características Diferenciales entre Maltratadores y no Maltratadores: El Caso de la Psicopatología y el Consumo de Alcohol o Drogas. PSYKHE. 2004; 13: 141-56.

25. Dutton D. MCMI Results for Batterers: A Response to Gondolf. Journal of Family Violence. 2003; 18: 253-5.

26. Huss M, Langhinrichsen-Rohling J. Assessing the generalization of psychopathy in a clinical sample of domestic violence perpetrators. Law Hum Behav. 2006; 30: 571-86.

27. Swogger MT, Walsh Z, Kosson DS. Domestic violence and psychopathic traits: distinguishing the antisocial batterer from other antisocial offenders. Aggress Behav. 2007; 33: 253-60.

28. Gondolf EW. Characteristics of Batterers in a Multi-site Evaluation of Batterer Intervention Systems. 1996[acceso 20 de marzo de 2008]. Disponible en http: //www.mincava.umn.edu/ documents/gondolf/batchar.html

29. Tilley DS, Brackley M. Men who batter intimate partners: a grounded theory study of the development of male violence in intimate partner relationships. Issues Ment Health Nurs. 2005; 26: 281-97.

30. Easton CJ, Mandel DL, Hunkele, KA, Nich C, Rounsaville, BJ, Carroll KM. A Cognitive Behavioral Therapy for Alcohol-dependent Domestic Violence Offenders: An Integrated Substance Abuse-Domestic Violence Treatment Approach (SADV). The American Journal on Addictions, 2007; 16: 24-31.

31. Fals-Stewart W. The occurrence of partner physical aggression on days of alcohol consumption: a longitudinal diary study. J Consult Clin Psych. 2003; 71: 41-52.

32. Sharps PW, Campbell J, Campbell D, Faye G, Webster D. The role of alcohol use in intimate partner femicide. Am J Addict. 2001; 10: 122-35.
33. Logan TK, Walker R, Staton M, Lenkefeld C. Substance Use and Intimate Violence Among Incarcerated Males. Journal of Family Violence 2001; 16: 93-114.

34. Corvo K, Halpern J, Ferraro FR. Frontal Lobe Deficits and Alcohol Abuse: Possible Interactions in Predicting Domestic Violence. Journal of Aggression, Maltreatment \& Trauma 2006; 3: 49-63.

35. Rosenbaum A, Hoge SK, Adelman SA, Warnken WJ, Fletcher KE, Kane RL. Head Injury in Partner-Abusive Men. J Consult Clin Psychol. 1994 Dec; 62: 1187-93

36. Teichner G, Golden CJ, Van Hasselt VB, Peterson A. Assessment of cognitive functioning in men who batter. Int J Neurosci. 2001; 111: 241-53.

37. Calvete E. Justification of Violence and Grandiosity Schemas as Predictors of Antisocial Behavior in Adolescents. J Abnorm Child Psychol. En prensa.

38. Arroyo JM, Ortega E. Un programa de mejora de la calidad asistencial de los problemas de salud mental en prisión. Evaluación de resultados después de 6 años (2000-2005) Rev Esp Sanid Penit. 2007; 9: 11-20.

39. López-Barrachina R, Lafuente O, García-Latas JL. Del mito de Narciso a los trastornos de la personalidad en las cárceles aragonesas: Una aproximación al perfil de estos desórdenes en las personas privadas de libertad. Rev Esp Sanid Penit. 2007; 9: 53-63

40. Sellbom M, Ben-Porath YS, Baum LJ, Erez E, Gregory C. Predictive validity of the MMPI-2 Restructured Clinical (RC) Scales in a batterers' intervention program. J Pers Assess. 2008; 90: 129-35.

41. Bowen E, Gilchrist E. Predicting dropout of court-mandated treatment in a British sample of domestic violence offenders. Int J Offender Ther Comp Criminol. 2004; 48: 215-34.

42. Lawson DM, Dawson TE, Kieffer KM, Perez LM, Burke J, Kier FJ. An integrated feminist/cognitivebehavioral and psychodynamic group treatment model for men who abuse their partners. Psychology of Men and Masculinity, 2001; 2: 86-99.

\section{CORRESPONDENCIA}

Esther Calvete

Departamento de Psicología

Universidad de Deusto

Apdo. 1, 48080 Bilbao

Teléfono: 944139000 (Ext. 2228)

email: ecalvete@fice.deusto.es 